\title{
Solvability, Completeness and Computational Analysis of A Perturbed Control Problem with Delays
}

\author{
Ludwik Byszewski $^{1}$, Denis Blackmore ${ }^{2, *}$, Alexander A. Balinsky ${ }^{3}$, \\ Anatolij K. Prykarpatski ${ }^{1,4}$ and Mirosław Luśtyk ${ }^{4}$ \\ ${ }^{1}$ Institute of Mathematics at Cracow University of Technology, ul.Warszawska, Poland \\ ${ }^{2}$ Department of Mathematical Sciences at NJIT, University Heights, Newark NJ 07102, USA \\ ${ }^{3}$ Mathematics Institute,Cardiff University, Cardiff CF24 4AG, Great Britain \\ ${ }^{4}$ Department of Applied Mathematics,AGH University of Technology, Cracow 30-059, Poland
}

Received November 1, 2019; Revised December 25, 2019; Accepted December 30, 2019

Copyright $@ 2020$ by authors, all rights reserved. Authors agree that this article remains permanently open access under the terms of the Creative Commons Attribution License 4.0 International License

\begin{abstract}
As a first step, we provide a precise mathematical framework for the class of control problems with delays (which we refer to as the control problem) under investigation in a Banach space setting, followed by careful definitions of the key properties to be analyzed such as solvability and complete controllability. Then, we recast the control problem in a reduced form that is especially amenable to the innovative analytical approach that we employ. We then study in depth the solvability and completeness of the (reduced) nonlinearly perturbed linear control problem with delay parameters. The main tool in our approach is the use of a Borsuk-Ulam type fixed point theorem to analyze the topological structure of a suitably reduced control problem solution, with a focus on estimating the dimension of the corresponding solution set, and proving its completeness. Next, we investigate its analytical solvability under some special, mildly restrictive, conditions imposed on the linear control and nonlinear functional perturbation. Then, we describe a novel computational projection-based discretization scheme of our own devising for obtaining accurate approximate solutions of the control problem along with useful error estimates. The scheme effectively reduces the infinite-dimensional problem to a sequence of solvable finite-dimensional matrix valued tasks. Finally, we include an application of the scheme to a special degenerate case of the problem wherein the Banach-Steinhaus theorem is brought to bear in the estimation process.
\end{abstract}

Keywords Perturbed Linear Control Problem, Delay, Solvability, Stability, Computational Scheme, Convergence

\section{Introduction}

Linear control problems with nonlinear functional perturbations are of great interest in modern studies owing to their nontrivial mathematical structure and wide applications in diverse fields. Of special interest are nonlinearly perturbed control problems $[11,25,26]$ with delay parameters, modeling some real situations in widely used remote control systems. The solvability and reliability of such control problems strongly depends on the topological structure $[1,3,4,8,10,11,17,15,23]$ of the corresponding solution set, its completeness and stability. Here we study in detail the solvability, completeness and topological structure of the corresponding solution set for a suitably reduced linear control problem with nonlinear functional perturbation, depending on delay parameters, using generalized fixed point $[9,18,19,20,21,22]$ theory, which enabled us to advance the body of knowledge found in the literature. We investigate its classical analytical solvability under some special nonlocal conditions $[2,7]$, imposed on the linear control and nonlinear functional perturbation, and study the feasibility of a naturally related control problem computational scheme, based on the classical projection discretization method, which reduces the infinite dimensional problem to a sequence of solvable finite dimensional matrix valued tasks. The solvability and completeness of the (reduced) nonlinearly perturbed linear control problem with delay parameters is studied in detail making use of a Borsuk-Ulam type fixed 
point theorem that is particularly well-suited for analyzing the general topological structure of certain kinds of reduced control problem solutions, with a focus on estimating the dimension of the corresponding solution set, and proving its completeness. We also investigate the related classical analytical solvability under some special, mildly restrictive, conditions imposed on the linear control and nonlinear functional perturbation. As its application oriented part there is described a novel computational projection-based discretization scheme for obtaining accurate approximate solutions of the control problem along with useful error estimates. The numerical scheme effectively reduces infinite-dimensional problem to a sequence of solvable finite dimensional matrix valued tasks. In addition, we include an application of the scheme to a special degenerate case of the problem wherein the Banach-Steinhaus theorem is brought to bear in the estimation process.

\section{Control problem: controllability and solution set analysis}

We begin by describing our control as a mathematical problem $[5,11,13,24,26]$ posed in Banach spaces. Let $A C^{1}([0,1] ; X)$ be the space of absolutely continuous differentiable functions on the closed interval $[0,1] \subset \mathbb{R}$, taking values in a Banach space $X, L_{\infty}([0,1] ; Y)$ be the space of essentially bounded functions on $[0,1] \subset \mathbb{R}$ with values in a Banach space $Y$, and $L_{1}([0,1] ; X)$ be the space of integrable functions on $[0,1] \subset \mathbb{R}$ with values in the Banach space $X$.

Consider the following second-order evolution control problem:

$$
\ddot{x}-A(t) x-B(t) u=f\left(t ; u, x, x \circ a_{0}, \dot{x}, \dot{x} \circ a_{1}\right)
$$

with boundary conditions:

$$
\begin{aligned}
& x(0)=x_{0} \in X, \dot{x}(0)=x_{0}^{\prime} \in X, \\
& x(1)=x_{1} \in X, \quad u \in U,
\end{aligned}
$$

for some control subset $U \subset L_{\infty}([0,1] ; Y)$, where $\dot{x}:=d x / d t, x \circ a_{0}(t):=x\left(t-a_{0}(t)\right)$ and $\dot{x} \circ a_{1}(t):=\dot{x}\left(t-a_{1}(t)\right)$ for positive delay functions $a_{0}, a_{1}:[0,1] \rightarrow[0,1]$, satisfying the conditions $a_{0}(t)-t \leq 0, a_{1}(t)-t \leq 0$ for almost all $t \in[0,1]$, $A:[0,1] \rightarrow \mathcal{L}(X ; X)$ and $B:[0,1] \rightarrow \mathcal{L}(Y ; X)$ are linear integrable maps, and $f:[0,1] \times Y \times X^{4} \rightarrow X$ satisfies

$1^{0}$ for all $x, \dot{x} \in X$ and $u \in Y$ the map $f\left(\cdot, u ; x, x \circ a_{1}, \dot{x}, \dot{x} \circ a_{2}\right):[0,1] \rightarrow X$ is measurable;

$2^{0}$ for almost all $t \in[0,1]$ the map $f(t, \cdot ; \cdot): Y \times X^{4} \rightarrow X$ is continuous;

$3^{0}$ there exist positive integrable functions $\alpha, \beta:[0.1] \rightarrow \mathbb{R}_{+}$, such that the inequality $\left\|f\left(t ; u, x, x \circ a_{1}, \dot{x}, \dot{x} \circ a_{2}\right)\right\| \leq$ $\alpha(t)\left(\|u\|+\|x\|+\left\|x \circ a_{1}\right\|+\|\dot{x}\|+\left\|\dot{x} \circ a_{2}\right\|\right)+\beta(t)$ holds for all $u \in Y$, all $x, \dot{x}, x \circ a_{1}, \dot{x} \circ a_{2} \in X$ and almost all $t \in[0,1]$.

By a solution to the problem (2.1) - (2.2) we mean a pair $(x, u):[0,1] \rightarrow X \times Y$, such that the mapping $(x, u) \in$ $A C^{1}([0,1] ; X) \times L_{\infty}([0,1] ; Y)$ satisfies for almost all $t \in[0,1]$ the control equation (2.1) and the boundary conditions $(2.2)$.

Let $U \subset L_{\infty}([0,1] ; Y)$ be a suitable control subspace. Then we say that the dynamical system (2.1) - (2.2) is completely controllable on the subspace $U \subset L_{\infty}([0,1] ; Y)$, if it has a solution $x \in A C^{1}([0,1] ; X)$ and $u \in U$ for all $x_{0}, x_{0}^{\prime}$ and $x_{1} \in X$. Next, we study this controllability problem as that of describing the existence of the corresponding solution set of (2.1) - (2.2) and its topological structure.

The problem (2.1) - (2.2) can be recast as the reduced control system

$$
\begin{gathered}
\ddot{x}-A(t) x-B(t) u=f\left(t ; u, x, x \circ a_{0}, \dot{x}, \dot{x} \circ a_{1}\right), \\
\int_{0}^{1} S_{1}(1) S_{1}(t) B(t) u d t= \\
S_{0}(1) x_{0}+S_{1}(1) x_{0}^{\prime}-x_{1}-\int_{0}^{1} S_{1}(1) S_{1}(t) f\left(t ; u, x, x \circ a_{0}, \dot{x}, \dot{x} \circ a_{1}\right) d t,
\end{gathered}
$$

under the constraint, where $\left(S_{0}(t), S_{1}(t): X^{2} \rightarrow A C^{1}([0,1] ; X)\right.$ for almost all $t \in[0,1]$ is an operator solution to the uniform linear system

$$
\ddot{x}-A(t) x=0
$$


with Cauchy data

$$
x(0)=x_{0} \in X, \quad \dot{x}(0)=x_{0}^{\prime} \in X,
$$

for which

$$
x(t)=S_{0}(t) x_{0}+S_{1}(t) x_{0}^{\prime}
$$

for all $t \in[0,1]$.

Now, based on the representation (2.1a) - (2.1b), one can define a linear operator $\mathcal{A}: A C^{1}([0,1] ; X) \times L_{\infty}([0,1] ; Y) \rightarrow$ $L_{1}([0,1] ; Y) \times X$ via the expression

$$
\mathcal{A}(x, u)=\left(\ddot{x}-A(t) x-B(t) u, \int_{0}^{1} S_{1}(1) S_{1}(t) B(t) u d t\right),
$$

allowing to rewrite the control problem (2.1a) - (2.1b) as the following equivalent operator equation:

$$
\mathcal{A}(x, u)=\mathcal{F}(x, u)
$$

where $\mathcal{F}: A C^{1}([0,1] ; X) \times L_{\infty}([0,1] ; Y) \rightarrow L_{1}([0,1] ; Y) \times X$ is given by

$$
\begin{aligned}
\mathcal{F}(x, u) & :=\left(f\left(t ; u, x, x \circ a_{0}, \dot{x}, \dot{x} \circ a_{1}\right), S_{0}(1) x_{0}+S_{1}(1) x_{0}^{\prime}-x_{1}\right. \\
& \left.-\int_{0}^{1} S_{1}(1) S_{1}(t) f\left(t ; u, x, x \circ a_{0}, \dot{x}, \dot{x} \circ a_{1}\right) d t\right) .
\end{aligned}
$$

Consequently, we need to study the structure of the solution set $\mathcal{N}(\mathcal{A}, \mathcal{F}) \subset A C^{1}([0,1] ; X) \times L_{\infty}([0,1] ; Y)$ of the control problem (2.4), where the linear operator $\mathcal{A}: E_{1} \rightarrow E_{2}$ is assumed to be a closed, surjective mapping from the Banach space $E_{1}:=C^{1}([0,1] ; X) \times L_{\infty}([0,1] ; Y)$ onto the Banach space $E_{2}:=L_{1}([0,1] ; Y) \times X$ with naturally defined norms. The domain, $\operatorname{dom\mathcal {A}}:=A C^{1}([0,1] ; X) \times U \subset E_{1}$ should be chosen so that the control space $U \subset L_{\infty}([0,1] ; Y)$ satisfies the condition

$$
L_{\infty}([0,1] ; Y) \backslash \operatorname{ker} \mathcal{B} \subset U,
$$

where the linear operator $\mathcal{B}: L_{\infty}([0,1] ; Y) \rightarrow X$ acts as

$$
\mathcal{B} u:=\int_{0}^{1} S_{1}(1) S_{1}(t) f\left(t ; u, x, x \circ a_{0}, \dot{x}, \dot{x} \circ a_{1}\right) d t
$$

for any $u \in L_{\infty}([0,1] ; Y)$. Having assumed that the condition (2.7) holds, we easily obtain the result.

Proposition 2.1. The reduced control problem (2.4) is completely controllable .

Proof. The statement readily follows from the condition (2.6) and the second part of the expression (2.5), guaranteeing the existence of the nontrivial solution $\operatorname{set} \mathcal{N}(\mathcal{A}, \mathcal{F}) \subset A C^{1}([0,1] ; X) \times L_{\infty}([0,1] ; Y)$ for the control problem (2.4).

Consider now the mapping (2.5) and assume additionally that its domain $\operatorname{dom\mathcal {F}}=\operatorname{dom\mathcal {A}} \cap S_{r}(0)$, where $S_{r}(0) \subset E_{1}$ is a sphere of radius $r>0$, centered at $0 \in E_{1}$. We need the following $[9,18,11]$ useful definitions.

Definition 2.2. A mapping $\mathcal{F}: E_{1} \rightarrow E_{2}$ from a Banach space $E_{1}$ to a Banach space $E_{2}$ is called $\mathcal{A}$-compact subject to a linear operator $\mathcal{A}: E_{1} \rightarrow E_{2}$, if it is continuous and for any bounded sets $A_{1} \subset \operatorname{dom\mathcal {F}}$ and $A_{2} \subset E_{2}$ the set $\mathcal{F}\left(A_{1} \cap \mathcal{A}^{-1}\left(A_{2}\right)\right) \subset E_{2}$ is relatively compact in $E_{2}$ (the empty set $\varnothing$ is considered, by definition, compact).

Given a continuous nonlinear mapping $\mathcal{F}: E_{1} \rightarrow E_{2}, \operatorname{dom} \mathcal{F} \subset S_{r}(0)$, and a closed, surjective linear operator $\mathcal{A}: E_{1} \rightarrow E_{2}$, one can also define the following numerical characteristics:

$$
k_{\mathcal{F}}:=\sup _{e_{1} \in S_{r}(0)} \frac{1}{r}\left\|\mathcal{F}\left(e_{1}\right)\right\|_{2}
$$

and

$$
k(\mathcal{A}):=\sup _{e_{2} \in E_{2}} \frac{1}{\left\|e_{2}\right\|_{2}} \inf _{e_{1} \in \operatorname{dom} \mathcal{A}}\left\{\left\|e_{1}\right\|_{1}: \mathcal{A} e_{1}=e_{2}\right\},
$$

where $k(\mathcal{A}):=\left\|\widetilde{\mathcal{A}}^{-1}\right\|$ and the operator $\widetilde{\mathcal{A}}:=\left.\mathcal{A}\right|_{E_{1} / \operatorname{ker} \mathcal{A}}$ is an invertible continuous linear operator from the factor-space $E_{1} / \operatorname{ker} \mathcal{A}$ onto $E_{2}$. Introduce preliminarily the following definition. 
Definition 2.3. The topological dimension of a closed compact set $A \subset X$ is the number $\operatorname{dim} A:=\inf \left\{k \in \mathbb{Z}_{+}:\right.$the condition $\bigcap_{j=1}^{k+2} U_{\alpha_{j}}=\varnothing$ is satisfied for any subsets $U_{\alpha_{j}} \in\left\{U_{\alpha_{\beta}} \subset X\right\}$ of all specially chosen subcoverings $\left\{U_{\alpha_{\beta}} \subset X\right\}$ of any covering $\left\{U_{\alpha}\right\}$ of the set $\left.A\right\}$.

Then, using a generalized Borsuk-Ulam type fixed point theorem $[9,18]$, one can formulate the following result that characterizes the solution set $\mathcal{N}(\mathcal{A}, \mathcal{F}) \subset A C^{1}([0,1] ; X) \times L_{\infty}([0,1] ; Y)$ of the reduced control problem (2.4).

Theorem 2.4. Let the linear closed operator $\mathcal{A}: E_{1} \rightarrow E_{2}$, defined by the expression (2.3), satisfy the dimension condition $\operatorname{dim} \operatorname{ker} \mathcal{A} \geq 1$, the continuous mapping $\mathcal{F}: E_{1} \rightarrow E_{2}$, defined by the nonlinear expression (2.5), be $\mathcal{A}$-compact and the inequality $k(\mathcal{A})<k_{\mathcal{F}}$ hold. Then the reduced problem (2.4) is solvable in the dom $\mathcal{F} \subset S_{r}(0)$, with a nonempty solution set $\mathcal{N}(\mathcal{A}, \mathcal{F}) \subset A C^{1}([0,1] ; X) \times L_{\infty}([0,1] ; Y)$ with topological dimension $\operatorname{dim} \mathcal{N}(\mathcal{A}, \mathcal{F}) \geq \operatorname{dim} \operatorname{ker} \mathcal{A}-1$.

Using the above result, one can easily check that for the reduced control problem (2.4) and for any sphere $S_{r}(0) \subset E_{1}, r>0$, the required inequality $k(\mathcal{A})<k_{\mathcal{F}}$ holds under natural integral constraints on the functions $\alpha, \beta:[0,1] \rightarrow \mathbb{R}$ and the related norm of the operator $\mathcal{B}: L_{\infty}([0,1] ; Y) \rightarrow X, j=\overline{0,1}$, characterizing the control set $U \subset L_{\infty}([0,1] ; Y)$ and the related complete controllability of the problem (2.1)-(2.2) under investigation.

Taking the statement above into account, we will study the topological structure of the solution set $\mathcal{N}(\mathcal{A}, \mathcal{F}) \subset A C^{1}([0,1] ; X) \times$ $L_{\infty}([0,1] ; Y)$ of the reduced control problem (2.4), when the linear operators $A(t): X \rightarrow X$ and $B(t): Y \rightarrow X, t \in[0,1]$, are endowed with some additional analytical structure. Namely, we consider the case, when for the closed linear operator $A(t): X \rightarrow X, t \in[0,1]$, there exists a linear operator $C(t): X \rightarrow X$ such that

$$
\ddot{C}(t)=A(t)
$$

for almost all $t \in[0,1]$. Concerning the operator family $\{B(t): Y \rightarrow X: t \in[0,1]\}$, one assumes that the induced mapping $B(t): L_{\infty}([0,1] ; Y) \rightarrow L_{1}([0,1] ; X), t \in[0,1]$, is closed and $\operatorname{dom} B=U \subset L_{\infty}([0,1] ; Y)$ satisfies the complete controllability condition (2.6).

\section{Classical solution set analysis}

Consider a slightly generalized nonlocal evolution control problem (2.1) in the following form:

$$
x^{\prime \prime}(t)-A x(t)-B(t) u(t-h)=f\left(t, u(t), x(t), x\left(a_{1}(t)\right), x^{\prime}(t), x^{\prime}\left(a_{2}(t)\right)\right), t \in(0, T],
$$

where boundary conditions are chosen in following functional form:

$$
\begin{gathered}
x(0)+g(x)=x_{0}, \\
x^{\prime}(0)+k(x)=x_{1},
\end{gathered}
$$

and

$$
u(t)=u_{0}(t), t \in[-h, 0),
$$

modifying those in (2.1a) - (2.1b), where $A$ is a linear operator from a real Banach space $X$ into itself, $B:[0, T] \longrightarrow \mathcal{L}(Y ; X)$, where $Y$ is a real Banach space, $f:[0, T] \times Y \times X^{4} \longrightarrow X, g: \mathcal{C}^{1}([0, T], X) \longrightarrow X, k: \mathcal{C}^{1}([0, T], X) \longrightarrow X, a_{i}:[0, T] \longrightarrow$ $[0, T], a_{i}(t) \leqslant t(i=1,2), x:[0, T] \longrightarrow X, u(t) \in Y$ for all admissible $t$ and $x_{0}, x_{1} \in X$.

We prove two theorems on the controllability of problem (3.1) - (3.4). In particular, we will show that the corresponding solution set to the problem (3.1) - (3.4) is nonempty and has a nontrivial topological dimension on the control space $U$, thus proving the problem controllability. For this purpose we apply the adapted controllability of semilinear control systems of the first order with constant time-delay equation control in [16] and the classical Banach contraction mapping theorem.

In Section 3, we use the following assumption:

Assumption $(A)$. Operator $A$ is the infinitesimal generator of a strongly continuous cosine family $\{C(t): t \in \mathbb{R}\}$ of bounded linear operators from $X$ into itself.

Recall that the infinitesimal generator of a strongly continuous cosine family $C(t)$ is the operator $A: X \supset D(A) \rightarrow X$ defined by

$$
A x:=\left.\frac{d^{2}}{d t^{2}} C(t) x\right|_{t=0}, x \in D(A),
$$

where

$$
D(A):=\left\{x \in X: C(t) x \text { is of class } \mathcal{C}^{2} \text { with respect to } t\right\} .
$$


Let

$$
E:=\left\{x \in X: C(t) x \text { is of class } \mathcal{C}^{1} \text { with respect to } t\right\} .
$$

The associated sine family $\{S(t): t \in \mathbb{R}\}$ is defined by the integral expression

$$
S(t) x:=\int_{0}^{t} C(s) x d s, \quad x \in X, t \in \mathbb{R} .
$$

Following $[6,16]$, we can derive that the mild solution $x(\cdot)$ to the system (3.1) - (3.4) satisfying the equation

$$
\begin{aligned}
& x(t)=C(t) x_{0}+S(t) x_{1}-C(t) g(x)-S(t) k(x)+\int_{0}^{t} S(t-s) B(s) u(s-h) d s \\
& +\int_{0}^{t} S(t-s) f\left(s, u(s), x(s), x\left(a_{1}(s)\right), x^{\prime}(s), x^{\prime}\left(a_{2}(s)\right)\right) d s \\
& =C(t) x_{0}+S(t)\left(x_{1}+b_{0}\right)-C(t) g(x)-S(t) k(x)+\int_{0}^{t-h} S(t-s+h) B(s+h) u(s) d s \\
& +\int_{0}^{t} S(t-s) f\left(s, u(s), x(s), x\left(a_{1}(s)\right), x^{\prime}(s), x^{\prime}\left(a_{2}(s)\right)\right) d s, t \in[0, T],
\end{aligned}
$$

where

$$
b_{0}:=\int_{-h}^{0} S(t-s+h) B(s+h) u_{0}(s) d s .
$$

Moreover, it is clear that the function $x(\cdot)$ belongs to the class $\mathcal{C}^{1}([0, T], X)$.

We shall apply the notation

$$
\begin{aligned}
& M_{1}:=\sup _{t \in[0, T]}\left\{\|C(t)\|+\|S(t)\|+\left\|S^{\prime}(t)\right\|\right\}, \\
& M_{2}:=\sup _{t \in[0, T]}\left\|\int_{0}^{t-h} S(t-s+h) B(s+h)[S(T-s+h) B(s+h)]^{*} d s\right\|, \\
& M_{3}:=\sup _{t \in[0, T]}\left\|[S(T-t+h) B(t+h)]^{*}\right\|,
\end{aligned}
$$

where * denotes the adjoint.

Moreover, let $B_{1}:=\mathcal{C}^{1}([0, T], X)$ and $B_{2}:=\mathcal{C}([0, T], Y)$.

Now, we define a real Banach space by

$$
\mathfrak{X}:=B_{1} \times B_{2}=\left\{(x, u): x \in B_{1}, u \in B_{2}\right\},
$$

endowed with the norm

$$
\|(x, u)\|_{\mathfrak{X}}:=\|x\|_{B_{1}}+\|u\|_{B_{2}} .
$$

Moreover, we define an operator $\mathcal{F}: \mathfrak{X} \longrightarrow \mathfrak{X}$ by

$$
\mathcal{F}(x, u):=\left(F_{1}(x, u), F_{2}(x, u)\right)=(y, v),
$$

where $F_{1}: \mathfrak{X} \longrightarrow B_{1}$ is given as

$$
\begin{aligned}
& F_{1}(x, u)(t)=y(t)=C(t) x_{0}+S(t)\left(x_{1}+b_{0}\right)-C(t) g(x)-S(t) k(x) \\
& +\left\{\int_{0}^{t-h} S(t-s+h) B(s+h)[S(T-s+h) B(s+h)]^{*} d s\right\} W^{-1} \mathcal{L}(x, u) \\
& +\int_{0}^{t} S(t-s) f\left(s, u(s), x(s), x\left(a_{1}(s)\right), x^{\prime}(s), x^{\prime}\left(a_{2}(s)\right)\right) d s, t \in[0, T]
\end{aligned}
$$


$F_{2}: \mathfrak{X} \longrightarrow B_{2}$ is defined by

$$
\begin{gathered}
F_{2}(x, u)(t)=v(t):=\left\{\begin{array}{cc}
{[S(T-t+h) B(t+h)]^{*} W^{-1} \mathcal{L}(x, u),} & t \in[0, T-h], \\
0, & t \in(T-h, T],
\end{array}\right. \\
W=W(T)=\int_{0}^{T-h} S(T-s+h) B(s+h)[S(T-s+h) B(s+h)]^{*} d s
\end{gathered}
$$

and

$$
\begin{aligned}
& \mathcal{L}(x, u)=x_{T}-C(T) x_{0}-S(T)\left(x_{1}+b_{0}\right)+C(T) g(x)+S(T) k(x) \\
& -\int_{0}^{T} S(T-s) f\left(s, u(s), x(s), x\left(a_{1}(s)\right), x^{\prime}(s), x^{\prime}\left(a_{2}(s)\right)\right) d s .
\end{aligned}
$$

Definition 3.1. The system (3.1) - (3.4) is said to be controllable over $X$ on $[0, T]$, if for every pair $\left(x_{0}, x_{T}\right) \in E \times X, x_{1} \in X$ and for every $u_{0}(\cdot) \in \mathcal{C}([-h, 0], Y)$ there exists at least one control function $u(\cdot) \in B_{2}$ such that with this control function on $[0, T]$, the corresponding mild solution $x(\cdot)$ to system (3.1) - (3.4) satisfies the condition $x(T)=x_{T}$.

To prove the controllability of system (3.1) - (3.4) we need the following result.

Theorem 3.2. The system (3.1) - (3.4) is controllable over $X$ on $[0, T]$ if and only if for every initial state $x_{0} \in E$ and a final state $x_{T} \in X$, the operator $\mathcal{F}: \mathfrak{X} \longrightarrow \mathfrak{X}$ given by (3.6) - (3.9) has a fixed point, i.e. there is some $(x, u) \in \mathfrak{X}$ such that $\mathcal{F}(x, u)=(x, u)$.

Proof. Let the system (3.1) - (3.4) be controllable. Then there exists a control function $u(\cdot) \in B_{2}$, which steers the state of the system given in equation (3.5) from $x_{0}$ to $x_{T}$. That is

$$
\begin{aligned}
& x_{\mathcal{T}} C(T) x_{0}+S(T)\left(x_{1}+b_{0}\right)-C(T) g(x)-S(T) k(x)+\int_{0}^{T-h} S(T-s+h) B(s+h) u(s) d s \\
& +\int_{0}^{T} S(T-s) f\left(s, u(s), x(s), x\left(a_{1}(s)\right), x^{\prime}(s), x^{\prime}\left(a_{2}(s)\right)\right) d s .
\end{aligned}
$$

From the above equation and from (3.9), we obtain

$$
\mathcal{L}(x, u)=\int_{0}^{T-h} S(T-s+h) B(s+h) u(s) d s .
$$

We then choose a function $u(\cdot)$ satisfying (3.10) as

$$
u(t):=\left\{\begin{array}{cc}
{[S(T-t+h) B(t+h)]^{*} W^{-1} \mathcal{L}(x, u),} & t \in[0, T-h], \\
0, & t \in(T-h, T] .
\end{array}\right.
$$

Now, if we compare (3.11) with (3.8), we see that $F_{2}(x, u)=u$. Moreover, with this control function, the corresponding solution given in (3.5) reduces to equation (3.7). Consequently, $F_{1}(x, u)=x$. Therefore, $\mathcal{F}(x, u)=(x, u)$, i.e. $\mathcal{F}$ has a fixed point.

Conversely, assume now that the operator $\mathcal{F}$ has a fixed point, i.e., $\mathcal{F}(x, u)=(x, u)$ for some $(x, u) \in \mathfrak{X}$. We want to show that there exists some control function $u(\cdot) \in B_{2}$ such that $x(T)=x_{T}$. Since $\mathcal{F}(x, u)=(x, u)$ then, by (3.7) and (3.8), we obtain the formulas

$$
\begin{aligned}
& x(t)=C(t) x_{0}+S(t)\left(x_{1}+b_{0}\right)-C(t) g(x)-S(t) k(x) \\
& +\left\{\int_{0}^{t-h} S(t-s+h) B(s+h)[S(T-s+h) B(s+h)]^{*} d s\right\} W^{-1} \mathcal{L}(x, u) \\
& +\int_{0}^{t} S(t-s) f\left(s, u(s), x(s), x\left(a_{1}(s)\right), x^{\prime}(s), x^{\prime}\left(a_{2}(s)\right)\right) d s
\end{aligned}
$$


and

$$
u(t)=\left\{\begin{array}{cl}
{[S(T-t+h) B(t+h)]^{*} W^{-1} \mathcal{L}(x, u),} & t \in[0, T-h], \\
0, & t \in(T-h, T] .
\end{array}\right.
$$

In order to get $x(T)=x_{T}$, we put $t=T$ in (3.12) and apply (3.9). Consequently, we have

$$
\begin{aligned}
& x(T)=x_{T}-\mathcal{L}(x, u)+\left\{\int_{0}^{T-h} S(T-s+h) B(s+h)[S(T-s+h) B(s+h)]^{*} d s\right\} W^{-1} \mathcal{L}(x, u) \\
& =x_{T}-\mathcal{L}(x, u)+W W^{-1} \mathcal{L}(x, u)=x_{T} .
\end{aligned}
$$

Moreover, problem (3.1) - (3.4) is controllable on $[0, T]$, so the proof of Theorem 3.2 is complete.

As a consequence, we show the nontriviality of the topological dimension of the corresponding solution set $\mathcal{N}(\mathcal{A}, \mathcal{F})$.

\section{Theorem 3.3. Suppose that:}

(i) Assumption (A) is satisfied,

(ii) $a_{i}:[0, T] \longrightarrow[0, T](i=1,2)$ are continuous on $[0, T], f:[0, T] \times Y \times X^{4} \longrightarrow X$ is continuous with respect to the first variable $t \in[0, T]$ and there exists positive constants $L, K_{1}, K_{2}$ such that

$$
\left\|f\left(s, w, z_{1}, z_{2}, z_{3}, z_{4}\right)-f\left(s, \tilde{w}, \tilde{z_{1}}, \tilde{z_{2}}, \tilde{z_{3}}, \tilde{z_{4}}\right)\right\|_{X} \leqslant L\left(\|w-\tilde{w}\|_{Y}+\sum_{i=1}^{4}\left\|z_{i}-\tilde{z_{i}}\right\|_{X}\right)
$$

for $s \in[0, T], w, \tilde{w} \in Y, z_{i}, \tilde{z}_{i} \in X(i=1,2,3,4)$,

$$
\left\|g\left(y_{1}\right)-g\left(y_{2}\right)\right\|_{X} \leqslant K_{1}\left\|y_{1}-y_{2}\right\|_{B_{1}}
$$

and

$$
\left\|k\left(y_{1}\right)-k\left(y_{2}\right)\right\|_{X} \leqslant K_{2}\left\|y_{1}-y_{2}\right\|_{B_{1}}
$$

(iii) $\max \left\{4 M_{1}\left[\left(K_{1}+K_{2}+2 T L\right)\left(1+M_{2}\left\|W^{-1}\right\|\right)\right], 2 M_{1} M_{3}\left\|W^{-1}\right\|\left(K_{1}+K_{2}+2 T L\right)\right\}<1$,

(iv) $x_{0} \in E$ and $x_{1} \in X$.

Then system (3.1) - (3.4) is controllable on $[0, T]$.

Proof. We will prove that the operator $\mathcal{F}$ given by (3.6) - (3.9) has a unique fixed point by using the Banach contraction principle, and the controllability follows from Theorem 3.2. Observe that

$$
\left\|F_{1}\left(y_{1}, v_{1}\right)-F_{1}\left(y_{2}, v_{2}\right)\right\|_{B_{1}}=\sup _{t \in[0, T]}\left\{\left\|F_{1}\left(y_{1}, v_{1}\right)(t)-F_{1}\left(y_{2}, v_{2}\right)(t)\right\|+\left\|F_{1}^{\prime}\left(y_{1}, v_{1}\right)(t)-F_{1}^{\prime}\left(y_{2}, v_{2}\right)(t)\right\|\right\}
$$

It is easy to see that

$$
\begin{aligned}
& \left\|F_{1}\left(y_{1}, v_{1}\right)(t)-F_{1}\left(y_{2}, v_{2}\right)(t)\right\| \leqslant \sup _{t \in[0, T]} \|-C(t)\left(g\left(y_{1}\right)-g\left(y_{2}\right)\right)-S(t)\left(k\left(y_{1}\right)-k\left(y_{2}\right)\right) \\
& +\left\{\int_{0}^{t-h} S(t-s+h) B(s+h)[S(T-s+h) B(s+h)]^{*} d s\right\} W^{-1}\left(\mathcal{L}\left(y_{1}, v_{1}\right)-\mathcal{L}\left(y_{2}, v_{2}\right)\right) \\
& +\int_{0}^{t} S(t-s)\left(f\left(s, v_{1}(s), y_{1}(s), y_{1}\left(a_{1}(s)\right), y_{1}^{\prime}(s), y_{1}^{\prime}\left(a_{2}(s)\right)\right)\right. \\
& \left.-f\left(s, v_{2}(s), y_{2}(s), y_{2}\left(a_{1}(s)\right), y_{2}^{\prime}(s), y_{2}^{\prime}\left(a_{2}(s)\right)\right)\right) d s \|_{1} \\
& \leqslant M_{1}\left(K_{1}+K_{2}\right)\left\|y_{1}-y_{2}\right\|_{B_{1}}+2 M_{1} T L\left(\left\|y_{1}-y_{2}\right\|_{B_{1}}+\left\|v_{1}-v_{2}\right\|_{B_{2}}\right) \\
& +M_{2}\left\|W^{-1}\right\|\left[M_{1}\left(K_{1}+K_{2}\right)\left\|y_{1}-y_{2}\right\|_{B_{1}}+2 M_{1} T L\left(\left\|y_{1}-y_{2}\right\|_{B_{1}}+\left\|v_{1}-v_{2}\right\|_{B_{2}}\right)\right] \\
& \leqslant \frac{\kappa_{1}}{4}\left\|\left(y_{1}, v_{1}\right)-\left(y_{2}, v_{2}\right)\right\|_{\mathfrak{X}},
\end{aligned}
$$

where

$$
\kappa_{1}:=4 M_{1}\left[\left(K_{1}+K_{2}+2 T L\right)\left(1+M_{2}\left\|W^{-1}\right\|\right)\right]
$$


Analogously,

$$
\left\|F_{1}^{\prime}\left(y_{1}, v_{1}\right)(t)-F_{1}^{\prime}\left(y_{2}, v_{2}\right)(t)\right\| \leqslant \frac{\kappa_{1}}{4}\left\|\left(y_{1}, v_{1}\right)-\left(y_{2}, v_{2}\right)\right\|_{\mathfrak{X}}
$$

Consequently

$$
\left\|F_{1}\left(y_{1}, v_{1}\right)-F_{1}\left(y_{2}, v_{2}\right)\right\|_{B_{1}} \leqslant \frac{\kappa_{1}}{2}\left\|\left(y_{1}, v_{1}\right)-\left(y_{2}, v_{2}\right)\right\|_{\mathfrak{X}}
$$

Also, observe that

$$
\begin{aligned}
& \left\|F_{2}\left(y_{1}, v_{1}\right)-F_{2}\left(y_{2}, v_{2}\right)\right\|_{B_{2}}=\sup _{t \in[0, T]}\left\|F_{2}\left(y_{1}, v_{1}\right)(t)-F_{2}\left(y_{2}, v_{2}\right)(t)\right\| \\
& =\sup _{t \in[0, T]}\left\|[S(T-t+h) B(t+h)]^{*} W^{-1}\left(\mathcal{L}\left(y_{1}, v_{1}\right)-\mathcal{L}\left(y_{2}, v_{2}\right)\right)\right\| \\
& \leqslant M_{3}\left\|W^{-1}\right\|\left(M_{1}\left(K_{1}+K_{2}\right)\left\|y_{1}-y_{2}\right\|_{B_{1}}+M_{1} T \sup _{s \in[0, T]} \| f\left(s, v_{1}(s), y_{1}(s), y_{1}\left(a_{1}(s)\right), y_{1}^{\prime}(s), y_{1}^{\prime}\left(a_{2}(s)\right)\right)\right. \\
& \left.-f\left(s, v_{2}(s), y_{2}(s), y_{2}\left(a_{1}(s)\right), y_{2}^{\prime}(s), y_{2}^{\prime}\left(a_{2}(s)\right)\right) \|\right) \\
& \left.\leqslant M_{1} M_{3}\left\|W^{-1}\right\|\left(K_{1}+K_{2}+2 T L\right)\left(\left\|y_{1}-y_{2}\right\|_{B_{1}}+\left\|v_{1}-v_{2}\right\|_{B_{2}}\right)\right]=\frac{\kappa_{2}}{2}\left\|\left(y_{1}, v_{1}\right)-\left(y_{2}, v_{2}\right)\right\|_{\mathfrak{X}},
\end{aligned}
$$

where

$$
\kappa_{2}:=2 M_{1} M_{3}\left\|W^{-1}\right\|\left(K_{1}+K_{2}+2 T L\right) .
$$

Therefore,

$$
\begin{aligned}
& \left\|\mathcal{F}\left(y_{1}, v_{1}\right)-\mathcal{F}\left(y_{2}, v_{2}\right)\right\|_{\mathfrak{X}}=\left\|F_{1}\left(y_{1}, v_{1}\right)-F_{1}\left(y_{2}, v_{2}\right)\right\|_{B_{1}}+\left\|F_{2}\left(y_{1}, v_{1}\right)-F_{2}\left(y_{2}, v_{2}\right)\right\|_{B_{2}} \\
& \leqslant \frac{\kappa_{1}}{2}\left\|\left(y_{1}, v_{1}\right)-\left(y_{2}, v_{2}\right)\right\|_{\mathfrak{X}}+\frac{\kappa_{2}}{2}\left\|\left(y_{1}, v_{1}\right)-\left(y_{2}, v_{2}\right)\right\|_{\mathfrak{X}} \leqslant \kappa\left\|\left(y_{1}, v_{1}\right)-\left(y_{2}, v_{2}\right)\right\|_{\mathfrak{X}},
\end{aligned}
$$

where

$$
\kappa:=\max \left\{\kappa_{1}, \kappa_{2}\right\} \in(0,1) .
$$

Consequently, $\mathcal{F}: \mathfrak{X} \longrightarrow \mathfrak{X}$ is a contraction mapping, so Banach's theorem implies that $\mathcal{F}$ has a unique fixed point in $\mathfrak{X}$. Hence, by Theorem 3.2, the system (3.1) - (3.4) is controllable on $[0, T]$, which completes the proof.

Remark 3.4. Let $p \in \mathbb{N}$ and $t_{1}, \ldots, t_{p}$ be given real numbers such that $0<t_{1}<\ldots<t_{p} \leqslant T$. Theorems 3.2 and 3.3 can be applied for $g$ defined by the formula

$$
\begin{gathered}
g(x)=\sum_{i=1}^{p} c_{i} x\left(t_{i}\right) \text { for } x \in C([0, T], X) \\
{\left[g(x)=\sum_{i=1}^{p} \frac{c_{i}}{\varepsilon_{i}} \int_{t_{i}-\varepsilon_{i}}^{t_{i}} x(s) d s \text { for } x \in C([0, T], X)\right],}
\end{gathered}
$$

where $c_{i}(i=1, \ldots, p)$ are given constants $\left[\right.$ and $\varepsilon_{i}(i=1, \ldots, p)$ are given positive constants such that $t_{i-1}<t_{i}-\varepsilon_{i}<t_{i}(i=$ $1, \ldots, p)$, respectively].

In particular, if $x_{0}=0, p=1, t_{1}=T, c:=-c_{1}\left[\varepsilon:=\varepsilon_{1}\right]$, then Theorems 3.2 and 3.3 are reduced to the theorems, where the nonlocal condition (3.2) is of the form

$$
x(0)=c x(T) \quad\left[x(0)=\frac{c}{\varepsilon} \int_{T-\varepsilon}^{T} x(s) d s\right],
$$

respectively. A similar remark is also true for the nonlocal condition (3.3).

\section{Computational Scheme and its Stability}

We are now interested in describing a feasible numerical computational scheme, based on the classical projection method and suitable for solving the nonlinear control problem, studied above. Consider the operator equation (2.4) and denote the related Banach space $A C^{1}([0,1] ; X) \times L_{\infty}([0,1] ; Y):=\mathcal{X}$ and the Banach space $L_{1}([0,1] ; Y) \times X:=\mathcal{Y}$. Then this operator equation can be rewritten as

$$
\mathcal{A}(w)=\mathcal{F}(w),
$$


where $w \in \mathcal{X}$ is its solution. Let now $\tilde{\mathcal{X}}_{N} \subset \tilde{\mathcal{X}}_{N+1} \subset \mathcal{X}$ and $\tilde{\mathcal{Y}}_{N} \subset \tilde{\mathcal{Y}}_{N+1} \subset Y, N \in \mathbb{Z}_{+}$, are suitable approximating finitedimensional Banach subspaces, $P_{N}^{(x)}: \mathcal{X} \rightarrow \tilde{\mathcal{X}}_{N}, N \in \mathbb{Z}_{+}$, and $P_{N}^{(y)}: \mathcal{Y} \rightarrow \tilde{\mathcal{Y}}_{N}, N \in \mathbb{Z}_{+}$, are the corresponding projectors. Now we consider a countable sequence of equations

$$
P_{N}^{(y)} \mathcal{A} \tilde{w}_{N}=P_{N}^{(y)} \mathcal{F}\left(\tilde{w}_{N}\right)
$$

on elements $\tilde{w}_{N} \in \tilde{\mathcal{X}}_{N}, N \in \mathbb{Z}_{+}$, which are suitable approximations to a searched solution of equation (4.1), being in general non-unique, as $\operatorname{dim} \operatorname{ker} \mathcal{A} \geq 1$. Note here that the projection method is called "realizable", if the set $\mathcal{M} \subset \mathcal{X}$ of solutions to equation (4.1) is nonempty, and for enough large $N \in \mathbb{Z}_{+}$there are nonempty sets $\mathcal{M}_{N} \subset \tilde{\mathcal{X}}_{N}$ of solutions to equations (4.2). The method is called "convergent" if it is realizable and there is fulfilled the condition

$$
\lim _{N \rightarrow \infty} \sup _{\tilde{w}_{N} \in \tilde{\mathcal{M}}_{N}} \inf _{w \in \mathcal{M}}\left\|\tilde{w}_{N}-w\right\|_{\mathcal{X}}=0
$$

It is obvious that for practical applications the realizability criteria of the projection method and its convergence are very important, therefore we will analyze them making use of modified version of Theorem 2.4. Namely, we assume that the necessary condition $\operatorname{dim} \operatorname{ker} \mathcal{A} \geq 1$ subject to the parameters of the whole control space $U$ is satisfied. Then the following result characterizes the realizability of the related computational scheme subject to the discrete approximations (4.2).

Theorem 4.1. Let a projector sequence $P_{N}^{(y)}: \mathcal{Y} \rightarrow \tilde{\mathcal{Y}}_{N}, N \in \mathbb{Z}_{+}$, satisfy the limiting condition

$$
\lim _{N \rightarrow \infty} \sup _{v \in \operatorname{Range}(\mathcal{A}) \cap \operatorname{Range}(\mathcal{F})}\left\|P_{N}^{(y)} v-v\right\|_{\mathcal{Y}}=0 .
$$

Then for sufficiently large integers $N \geq N_{0} \in \mathbb{Z}_{+}$the solution sets $\tilde{\mathcal{M}}_{N} \subset \tilde{\mathcal{X}}_{N}$ are nonempty and the convergence condition (4.3) holds.

Proof. Define

$$
\begin{gathered}
k_{\mathcal{F}}^{(N)}:=\sup _{\tilde{w}_{N} \in S_{r}(0)} \frac{1}{r}\left\|P_{N}^{(y)} \mathcal{F}\left(\tilde{w}_{N}\right)\right\|_{\mathcal{Y}_{N}}, \\
k_{\mathcal{A}}^{(N),-1}:=\sup _{\tilde{v}_{N} \in \tilde{\mathcal{Y}}_{N}} \frac{1}{\left\|\tilde{v}_{N}\right\|_{\tilde{\mathcal{Y}}_{N}}} \inf _{\tilde{w}_{N} \in P_{N}^{(x)} D(\mathcal{A})}\left\{\left\|\tilde{w}_{N}\right\|_{\tilde{\mathcal{X}}_{N}}: P_{N}^{(y)} \mathcal{A} \tilde{w}_{N}=\tilde{v}_{N}\right\},
\end{gathered}
$$

and choose such integer $N_{0} \in \mathbb{Z}_{+}$, that $\operatorname{dim} \operatorname{ker}\left(P_{N_{0}}^{(y)} \mathcal{A}\right) \geq 1$, and

$$
k_{\mathcal{F}} \leq k_{\mathcal{F}}^{\left(N_{0}\right)}<k_{\mathcal{A}}^{\left(N_{0}\right)} \leq k_{\mathcal{A}} .
$$

Then based on expressions (4.5) and (4.6) from condition (4.7) we obtain that for all $N \geq N_{0}$ the following inequalities

$$
k_{\mathcal{F}} \leq k_{\mathcal{F}}^{(N)}<k_{\mathcal{A}}^{(N)} \leq k_{\mathcal{A}}
$$

hold. This means that, owing to the generalized Leray-Schauder type fixed point theorem [18, 19, 20, 21, 22, 23], the sequence of equations (4.3) has solutions for all $N \geq N_{0}$, that is all solution sets $\tilde{\mathcal{M}}_{N} \subset \tilde{\mathcal{X}}_{N}, N \geq N_{0}$, are nonempty, and the projectionalgebraic method itself is realizable.

Take now $\varepsilon>0$ and consider the neighborhood

$$
U_{\varepsilon}(\mathcal{M}):=\left\{w \in D(\mathcal{F}): \inf _{\bar{w} \in \mathcal{M} \subset D(\mathcal{F})}\|\bar{w}-w\|_{\mathcal{X}}<\varepsilon .\right\}
$$

It is evident that the closed set $D(\mathcal{F}) \backslash U_{\varepsilon}(\mathcal{M})$ does not contain solutions to equation (4.1), and for some $\alpha_{\varepsilon}>0$ the inequality

$$
\inf _{w \in D(\mathcal{F}) \backslash U_{\varepsilon}(\mathcal{M})}\|\mathcal{A} w-\mathcal{F}(w)\|_{\mathcal{Y}}:=\alpha_{\varepsilon}>0
$$

holds. Choose now, based on (4.4), an integer $N_{\varepsilon} \in \mathbb{Z}_{+}$in such a way that for all $N \geq N_{\varepsilon}$

$$
\sup _{w \in D(\mathcal{F})}\left(\left\|\mathcal{A} w-P_{N}^{(y)} \mathcal{A} w\right\|_{\mathcal{Y}}+\left\|\mathcal{F}(w)-P_{N}^{(y)} \mathcal{F}(w)\right\|_{\mathcal{Y}}\right)<\alpha_{\varepsilon} .
$$

Then for all $w \in D(\mathcal{F}) \backslash U_{\varepsilon}(\mathcal{M})$ the following inequality

$$
\begin{gathered}
\left\|P_{N}^{(y)} \mathcal{A} w-P_{N}^{(y)} \mathcal{F}(w)\right\|_{\mathcal{Y}} \geq\|\mathcal{A} w-\mathcal{F}(w)\|_{\mathcal{Y}}- \\
-\left(\left\|\mathcal{A} w-P_{N}^{(y)} \mathcal{A} w\right\|_{\mathcal{Y}}+\left\|\mathcal{F}(w)-P_{N}^{(y)} \mathcal{F}(w)\right\|_{\mathcal{Y}}\right)>\alpha_{\varepsilon}-\alpha_{\varepsilon}=0,
\end{gathered}
$$

holds, that is for $N \geq N_{\varepsilon}$ there exists the imbedding $\tilde{\mathcal{M}}_{N} \subset U_{\varepsilon}(\mathcal{M})$. Since $\varepsilon>0$ is chosen enough small, the condition $\tilde{\mathcal{M}}_{N} \subset U_{\varepsilon}(\mathcal{M})$ for all $N \geq N_{\varepsilon}$ is equivalent to that of convergence for (4.3), proving the theorem. $\triangleright$ 
Remark 4.2. In the case when the sequences of $\tilde{\mathcal{X}}_{N} \subset \tilde{\mathcal{X}}_{N+1} \subset \mathcal{X}, N \in \mathbb{Z}_{+}$and $\tilde{\mathcal{Y}}_{N} \subset \tilde{\mathcal{Y}}_{N+1} \subset \mathcal{Y}, N \in \mathbb{Z}_{+}$, are Hilbert spaces and, moreover

$$
\cup_{N \in \mathbb{Z}_{+}} \tilde{\mathcal{X}}_{N}=\mathcal{X}, \quad \cup_{N \in \mathbb{Z}_{+}} \tilde{\mathcal{Y}}_{N}=\mathcal{Y}
$$

with projectors $P_{N}^{(x)}: \mathcal{X} \rightarrow \tilde{\mathcal{X}}_{N}, P_{N}^{(y)}: \mathcal{Y} \rightarrow \tilde{\mathcal{Y}}_{N}, N \in \mathbb{Z}_{+}$, being operators of orthogonal projection, the norms $\left\|P_{N}^{(x)}\right\|=1$, $\left\|P_{N}^{(x)}\right\|=1, N \in \mathbb{Z}_{+}$, and for all $w \in \mathcal{X}, v \in \mathcal{Y}$

$$
\lim _{N \rightarrow \infty}\left\|w-P_{N}^{(x)} w\right\|_{\mathcal{Y}}=0, \quad \lim _{N \rightarrow \infty}\left\|v-P_{N}^{(y)} v\right\|_{\mathcal{Y}}=0
$$

We assume further that conditions (4.3), (4.4) are fulfilled and $\operatorname{dim} \operatorname{ker} \mathcal{A} \geq 1$. Then an analog of Theorem 4.1 about the realizability of the projection computational scheme of discrete approximations for nonlinear operator equation (4.1) in Hilbert spaces holds.

Theorem 4.3. For sufficiently large $N \in \mathbb{Z}_{+}$solution sets $\tilde{\mathcal{M}}_{N} \subset \tilde{X}_{N}$ are nonempty and the convergence condition (4.2) holds.

Proof. It is clear that we need only to verify (4.4). Having assumed contrary, one can find such a subsequence of indices $N_{k} \in \mathbb{Z}_{+}$for $k \in \mathbb{Z}_{+}$, as well as elements $w_{k} \in D(\mathcal{F}), k \in \mathbb{Z}_{+}$, for which there exists $\varepsilon>0$, that

$$
\left\|P_{N_{k}}^{(y)} \mathcal{F}\left(w_{k}\right)-\mathcal{F}\left(w_{k}\right)\right\|_{\mathcal{Y}}>\varepsilon
$$

Since for all $k \in \mathbb{Z}_{+}$elements $f\left(w_{k}\right) \in \operatorname{Range}(\mathcal{A})$, owing to the $\mathcal{A}$-compactness of the mapping $f: D(\mathcal{F}) \rightarrow \mathcal{Y}$ there exists the $\operatorname{limit}_{k \rightarrow \infty} f\left(w_{k}\right)=\bar{w} \in \mathcal{Y}$. Making now use of the existence of limits (4.13), we obtain:

$$
\begin{gathered}
\lim _{k \rightarrow \infty}\left\|P_{N_{k}}^{(y)} \mathcal{F}\left(w_{k}\right)-\mathcal{F}\left(w_{k}\right)\right\|_{\mathcal{Y}} \leq \lim _{k \rightarrow \infty}\left\|P_{N_{k}}^{(y)} \mathcal{F}\left(w_{k}\right)-P_{N_{k}}^{(y)} \bar{w}\right\|_{\mathcal{Y}}+ \\
+\lim _{k \rightarrow \infty}\left\|P_{N_{k}}^{(y)} \bar{w}-\bar{w}\right\|_{\mathcal{Y}}+\lim _{k \rightarrow \infty}\left\|\bar{w}-\mathcal{F}\left(w_{k}\right)\right\|_{\mathcal{Y}}=0,
\end{gathered}
$$

contradicting the initial inequality (4.14), thereby proving the theorem. $\triangleright$

If the mapping $f: D(\mathcal{F}) \subset \mathcal{X} \rightarrow \mathcal{Y}$ is constant, the operator $\mathcal{A}: D(\mathcal{A}) \subset \mathcal{X} \rightarrow \mathcal{Y}$ is densely defined and $\overline{\operatorname{Range}(\mathcal{A}})=\mathcal{Y}$, one can prove additional convergence properties of the projection method of discrete approximations for equation (4.1), to which we proceed below.

\section{Computational scheme convergence analysis: a special degenerate case}

Consider the operator problem (4.1), when the mapping $\mathcal{F}: \mathcal{X} \rightarrow \mathcal{Y}$ is constant, that is $\mathcal{F}(w):=y \in \mathcal{Y}$ for all $w \in \mathcal{X}$. Assume that two families of finite-dimensional functional subspaces $\tilde{\mathcal{X}}_{N} \subset \mathcal{X}$ and $\tilde{\mathcal{Y}}_{N} \subset \mathcal{Y}$ for $N \in \mathbb{Z}_{+}$, are chosen such that

$$
\tilde{\mathcal{X}}_{N} \subset \tilde{\mathcal{X}}_{N+1}, \tilde{\mathcal{Y}}_{N} \subset \tilde{\mathcal{Y}}_{N+1}
$$

Assume that a region $\Omega \subset \mathbb{R}^{q}$ is bounded and has a sufficiently smooth boundary $\partial \Omega$, the space $\mathcal{X}:=L_{p}(\Omega ; \mathbb{R})$, the dense domain $D(A)=W_{p}^{(m+s)}(\Omega)$ and Range $(A)=W_{p}^{(s)}(\Omega) \subset L_{p}(\Omega ; \mathbb{R}):=Y, p>q, s>0$. The expressions

$$
\tilde{\mathcal{X}}_{N}:=P_{N}^{(x)} \mathcal{X}, \quad \tilde{\mathcal{Y}}_{N}:=P_{N}^{(y)} \mathcal{Y}
$$

where $P_{N}^{(x)}$ and $P_{N}^{(y)}$ are linear operators, defined naturally on continuous functions in the region $\Omega \subset \mathbb{R}^{q}$. Operators $P_{N}^{(x)}$ and $P_{N}^{(y)}$ are, evidently, projectors satisfying the conditions

$$
P_{N}^{(x)} P_{N}^{(x)}=P_{N}^{(x)}, \quad P_{N}^{(y)} P_{N}^{(y)}=P_{N}^{(y)}
$$

for all $N \in \mathbb{Z}_{+}$.

Consider now for each $N \in \mathbb{Z}_{+}$the following sequence of equations

$$
P_{N}^{(y)} \mathcal{A} \tilde{w}_{N}=P_{N}^{(y)} g
$$

on elements $\tilde{w}_{N} \in \tilde{\mathcal{X}}_{N}$, for which as $N \rightarrow \infty$

$$
\lim _{N \rightarrow \infty}\left\|A \tilde{w}_{N}-g\right\|_{\mathcal{Y}}=0
$$

where $g \in \mathcal{Y}$ is a fixed element of the space $\mathcal{Y}$. It is evident that equation (5.4) possesses a unique solution $\tilde{w}_{N} \in \tilde{\mathcal{X}}_{N}$, if for sufficiently large $N \in \mathbb{Z}_{+}$,

$$
P_{N}^{(y)} \mathcal{A} \tilde{X}_{N}=\tilde{Y}_{N}
$$


Condition (5.6) is equivalent to the existence of the inverse finite-dimensional operator of the mapping

$$
P_{N}^{(y)} \mathcal{A} P_{N}^{(x)}:=\mathcal{A}_{N}: \tilde{\mathcal{X}}_{N} \rightarrow \tilde{\mathcal{Y}}_{N}
$$

for large enough $N \in \mathbb{Z}_{+}$. $\mathcal{B}$

Next, we give a useful definition of an arbitrary limiting-dense family of subspaces $\left\{\mathcal{B}_{N} \subset \mathcal{B}: N \in \mathbb{Z}_{+}\right\}$of a Banach space

Definition 5.1. A family of subspaces $\left\{\mathcal{B}_{N} \subset \mathcal{B}: N \in \mathbb{Z}_{+}\right\}$is called limiting-dense in $\mathcal{B}$, if for each $g \in \mathcal{B}$ the following property

$$
\rho\left(g, \mathcal{B}_{N}\right):=\inf _{\tilde{w}_{N} \in \mathcal{B}_{N}}\left\|g-\tilde{w}_{N}\right\|_{\mathcal{B}} \rightarrow 0
$$

holds as $N \rightarrow \infty$.

For further analysis we will need the following convergence proposition for our approximation process.

Proposition 5.2. Let a linear operator $\mathcal{A}: \mathcal{X} \rightarrow \mathcal{Y}$ be invertible on a dense domain $D(\mathcal{A}) \subset \mathcal{X}$ and satisfy the condition $\overline{\text { Range }(\mathcal{A})}=\mathcal{Y}$, where $\mathcal{X}$ and $\mathcal{Y}$ are Banach spaces. Assume also that a family of subspaces $\left\{\mathcal{A} \tilde{\mathcal{X}}_{N} \in \mathcal{Y}: N \in \mathbb{Z}+\right\}$ is limiting-dense, and projection operators $P_{N}^{(y)}: \mathcal{Y} \rightarrow \tilde{\mathcal{Y}}_{N} \subset \mathcal{Y}$ satisfy the conditions

$$
\left\|P_{N}^{(y)}\right\| \leq c_{N}^{(y)}
$$

for some sequence $c_{N}^{(y)} \in \mathbb{R}_{+}, N \in \mathbb{Z}_{+}$. Then for any $g \in \mathcal{Y}$ a sequence of equations

$$
P_{N}^{(y)} \mathcal{A} w=g
$$

has the unique solutions $\tilde{w}_{N} \in \tilde{\mathcal{X}}_{N}$ for all $N \in \mathbb{Z}_{+}$, where

$$
\lim _{N \rightarrow \infty}\left\|\mathcal{A} \tilde{w}_{N}-g\right\|_{\mathcal{Y}}=0
$$

iff

i) condition (5.6) is satisfied;

ii) there exists such a positive sequence $\tau_{N}^{(y)} \in \mathbb{R}_{+}, N \in \mathbb{Z}_{+}$, that

$$
\left\|P_{N}^{(y)} \tilde{v}_{N}\right\|_{\tilde{\mathcal{Y}}_{N}} \geq \tau_{N}^{(y)}\left\|\tilde{v}_{N}\right\| \mathcal{Y}
$$

for each element $\tilde{v}_{N} \in A \tilde{\mathcal{X}}_{N}, N \in \mathbb{Z}_{+}$

iii) the upper limit

$$
\varlimsup_{N \rightarrow \infty}\left[\left(1+c_{N}^{(y)} \tau_{N}^{(y),-1}\right) \rho\left(g, \mathcal{A} \tilde{\mathcal{X}}_{N}\right)\right]=0
$$

for every $g \in Y$.

Proof. It is easy to see that the equation $P_{N}^{(y)} \mathcal{A} w=g, N \in \mathbb{Z}_{+}$, has solutions $\tilde{w}_{N} \in \tilde{\mathcal{X}}_{N}$, for which $\left\|\mathcal{A} \tilde{w}_{N}-g\right\|_{\mathcal{Y}} \rightarrow 0$ as $N \rightarrow \infty$. Then owing to the inequality

$$
\rho\left(g, \mathcal{A} \tilde{\mathcal{X}}_{N}\right)=\inf _{\tilde{w}_{N} \in \mathcal{A} \tilde{\mathcal{X}}_{N}}\left\|g-\mathcal{A} \tilde{w}_{N}\right\|_{\mathcal{Y}} \leq\left\|g-\mathcal{A} \tilde{w}_{N}\right\|_{\mathcal{Y}}
$$

one can infer that $\lim _{N \rightarrow \infty} \rho\left(g, \mathcal{A} \tilde{\mathcal{X}}_{N}\right)=0$, that is the family of subsets $\left\{A \tilde{\mathcal{X}}_{N} \in \mathcal{Y}: N \in \mathbb{Z}_{+}\right\}$is limiting-dense in $\mathcal{Y}$. Define now $N \in \mathbb{Z}_{+}$and consider $P_{N}^{(y)} \mathcal{A} w=\tilde{g}_{N} \in \tilde{\mathcal{Y}}_{N}$. It is clear that there exists such an element $g \in \mathcal{Y}$, for which $P_{N}^{(y)} g=\tilde{g}_{N}$, that is we obtained an equation which, owing to our assumptions of the Proposition 5.2, possesses the unique solution $\tilde{w}_{N} \in \tilde{\mathcal{X}}_{N}$. But this means that $P_{N}^{(y)} \mathcal{A} \tilde{\mathcal{X}}_{N}=\tilde{\mathcal{Y}}_{N}$, proving condition $i$ ) of our Proposition. Since the mapping $P_{N}^{(y)}: \mathcal{Y} \rightarrow \mathcal{Y}$ is a projector, then one can consider its constraint $\left.\bar{P}_{N}^{(y)}\right|_{A \tilde{\mathcal{X}}_{N}}: \mathcal{A} \tilde{\mathcal{X}}_{N} \rightarrow \tilde{\mathcal{Y}}_{N}$ for each $N \in \mathbb{Z}_{+}$. Operator $\bar{P}_{N}^{(y)}: \mathcal{A} \tilde{\mathcal{X}}_{N} \rightarrow \tilde{\mathcal{Y}}_{N}$ owing to (5.9) and (5.12) is bonded and one-to one mapping. Then based on the Banach theorem [12, 24, 4] about the inverse operator we obtain that there exists the inverse operator $\bar{P}_{N}^{(y),-1}: \tilde{\mathcal{Y}}_{N} \rightarrow \mathcal{A} \tilde{\mathcal{X}}_{N}$. 
Let now $\tilde{w}_{N} \in \tilde{\mathcal{X}}_{N}$ be the corresponding approximated solution of the equation $P_{N} \mathcal{A} w=P_{N} g$. Then the following equality $\mathcal{A} \tilde{w}_{N}=\bar{P}_{N}^{(y),-1} P_{N} g$ holds, from which and the condition (5.11) one obtains that

$$
\lim _{N \rightarrow \infty}\left\|\bar{P}_{N}^{(y),-1} P_{N} g-g\right\|_{\mathcal{Y}}=0
$$

for any $g \in \mathcal{Y}$. But this means that $\lim _{N \rightarrow \infty} \bar{P}_{N}^{(y),-1} P_{N} g=g$ for every given element $g \in \mathcal{Y}$. Making use of the classical Banach-Steinhaus theorem $[12,4,24]$ we obtain that

$$
\sup _{N \in \mathbb{Z}_{+}}\left\|\bar{P}_{N}^{(y),-1} P_{N}^{(y)}\right\| \mathcal{Y} \leq c^{(y)}<\infty
$$

for some bounded value $c^{(y)} \in \mathbb{R}_{+}$. Thus for each element $P_{N}^{(y)} \tilde{w}_{N}=\tilde{w}_{N}$ one finds that

$$
\left\|\bar{P}_{N}^{(y),-1} \tilde{w}_{N}\right\| \mathcal{Y}=\left\|\bar{P}_{N}^{(y),-1} P_{N}^{(y)} \tilde{w}_{N}\right\| \mathcal{Y} \leq\left\|\bar{P}_{N}^{(y),-1} P_{N}\right\| \mathcal{Y}\left\|j_{N} \tilde{w}_{N}\right\| \mathcal{Y} \leq c^{(y)}\left\|j_{N}\right\|\left\|\tilde{w}_{N}\right\| \mathcal{Y}
$$

where $j_{N}: \tilde{\mathcal{Y}}_{N} \rightarrow \mathcal{Y}$ is the corresponding densely defined imbedding operator and $\left\|j_{N}\right\|$ is its norm. Inequality (5.15) means that the norm of the operator $\bar{P}_{N}^{(y),-1}: \tilde{\mathcal{Y}}_{N} \rightarrow \mathcal{A} \tilde{\mathcal{X}}_{N} \subset \mathcal{Y}$ is for all $N \in \mathbb{Z}_{+}$uniformly bounded.

Choose now an arbitrary element $\tilde{v}_{N} \in \mathcal{A} \tilde{\mathcal{X}}_{N} \subset \mathcal{Y}$ and calculate $\tilde{w}_{N}:=\bar{P}_{N}^{(y)} \tilde{v}_{N} \in \tilde{\mathcal{Y}}_{N}$. Then making use of the inequality (5.15) we obtain

$$
\left\|\tilde{v}_{N}\right\|_{Y}=\left\|\bar{P}_{N}^{(y),-1} \tilde{w}_{N}\right\| \mathcal{Y} \leq\left\|\bar{P}_{N}^{(y),-1}\right\| \mathcal{Y}\left\|\tilde{w}_{N}\right\|_{\mathcal{Y}} \leq \tau_{N}^{(y),-1}\left\|P_{N} \tilde{v}_{N}\right\|_{\tilde{\mathcal{Y}}_{N}},
$$

where quantities $\tau_{N}^{(y)}>0$ are bounded for all $N \in \mathbb{Z}_{+}$. But this means that the condition $\left.i i\right)$ of our Statement is fulfilled concerning each element $\tilde{v}_{N} \in \mathcal{A} \tilde{\mathcal{X}}_{N}$, that is $\left\|P_{N}^{(y)} \tilde{v}_{N}\right\| \geq \tau_{N}^{(y)}\left\|\tilde{v}_{N}\right\| \mathcal{Y}, N \in \mathbb{Z}_{+}$.

Sufficiency of conditions $i$ ) - iii) shall next be proved as follows. Let us solve the equation $P_{N} \mathcal{A} w=P_{N} g$ for $N \in \mathbb{Z}_{+}$, whose $\tilde{w}_{N} \in \tilde{\mathcal{X}}_{N}$, whose solution is unique. Then they can be represented as

$$
\tilde{w}_{N}=\mathcal{A}^{-1} \bar{P}_{N}^{(y),-1} P_{N} g
$$

where, as above, the linear mapping $\bar{P}_{N}^{(y)}:=\left.P_{N}^{(y)}\right|_{\mathcal{A} \tilde{\mathcal{X}}_{N}}: \mathcal{A} \tilde{\mathcal{X}}_{N} \rightarrow \tilde{\mathcal{Y}}_{N}$ is the corresponding reduction upon $\mathcal{A} \tilde{\mathcal{X}}_{N} \subset \mathcal{Y}$ of the projection operator $P_{N}^{(y)}: \mathcal{Y} \rightarrow \mathcal{Y}$ upon the subspace $\tilde{\mathcal{Y}}_{N} \subset \mathcal{Y}$. Since, based on condition $\left.i i\right)$, we have $\left\|\bar{P}_{N}^{(y)}\right\| \mathcal{Y} \leq \tau_{N}^{(y),-1}$, the norm $\left\|\bar{P}_{N}^{(y),-1} P_{N}^{(y)}\right\| \leq c_{N}^{(y)} \tau_{N}^{(y),-1}$ for all $N \in \mathbb{Z}_{+}$. When for any element $\tilde{w}_{N} \in \mathcal{A} \tilde{\mathcal{X}}_{N}$ we obtain

$$
\begin{gathered}
\left\|\mathcal{A} \tilde{w}_{N}-g\right\|_{Y}=\left\|\bar{P}_{N}^{(y),-1} P_{N} g-g\right\|_{\mathcal{Y}} \leq \\
\leq \inf _{\tilde{w}_{N} \in \mathcal{A} \tilde{\mathcal{X}}_{N}}\left(\left\|\bar{P}_{N}^{(y),-1} P_{N} g-\bar{P}_{N}^{(y),-1} P_{N}^{(y)} \tilde{w}_{N}\right\|_{\mathcal{Y}}+\left\|\tilde{w}_{N}-g\right\|_{\mathcal{Y}}\right) \leq \\
\leq \inf _{\tilde{w}_{N} \in \mathcal{A} \tilde{\mathcal{X}}_{N}}\left(\left\|\bar{P}_{N}^{(y),-1} P_{N} g-\bar{P}_{N}^{(y),-1} P_{N}^{(y)} \tilde{w}_{N}\right\|_{\mathcal{Y}}+\left\|\tilde{w}_{N}-g\right\|_{\mathcal{Y}}\right) \leq \\
\leq \inf _{\tilde{w}_{N} \in \mathcal{A} \tilde{\mathcal{X}}_{N}}\left(c_{N}^{(y)} \tau_{N}^{(y),-1}+1\right) \rho\left(g, \tilde{w}_{N}\right)=\left(c^{(y)} \tau_{N}^{(y),-1}+1\right) \rho\left(g, \mathcal{A} \tilde{\mathcal{X}}_{N}\right),
\end{gathered}
$$

where we took into account that $\bar{P}_{N}^{(y),-1} P_{N}^{(y)} \tilde{w}_{N}=\tilde{w}_{N}$ for all $\tilde{w}_{N} \in \mathcal{A} \tilde{\mathcal{X}}_{N}$. But owing to the assumption $\left.i i i\right)$ this means the existence of the $\operatorname{limit}_{N \rightarrow \infty}\left\|\mathcal{A} \tilde{w}_{N}-g\right\|_{\mathcal{Y}}=0$ for an arbitrary element $g \in \mathcal{Y}$, finishing the proof. $\triangleright$

Remark 5.3. We note here that an analogous alternative of Proposition 5.2 was earlier proved in [14].

As an obvious corollary of the proof of Proposition 5.2 in the case when $\operatorname{dim} \tilde{\mathcal{X}}_{N}=\operatorname{dim} \tilde{\mathcal{Y}}_{N}<\infty$ for all $N \in \mathbb{Z}{ }_{+}$we obtain that condition $i$ ) in form (5.6) follows from $i i$ ). Moreover, the next statement about the convergence of the solutions $\tilde{w}_{N} \in \tilde{\mathcal{X}}_{N}$ as $N \rightarrow \infty$ to element $w \in \mathcal{X}$ holds.

Proposition 5.4. Let all the conditions of Proposition 5.2 be fulfilled, in particular, the invertible operator $\mathcal{A}: \mathcal{X} \rightarrow \mathcal{Y}$ is closed and surjective (this means that $\left\|\mathcal{A}^{-1}\right\|<\infty$ owing to the classical statement [12, 24, 4] about the closed everywhere defined operator). Then the sequence of solutions $\tilde{w}_{N} \in \tilde{\mathcal{X}}_{N}$ to the equation $P_{N}^{(y)} \mathcal{A} w=P_{N}^{(y)} g$, as $N \rightarrow \infty$, is the corresponding approximation to the solution of the equation $\mathcal{A} w=g$ subject to the norm $\|\cdot\|_{\mathcal{X}}$.

Proof. Assume that $w_{N} \in \mathcal{X}_{N}$ is a solution to the equation $P_{N}^{(y)} \mathcal{A} w_{N}=P_{N}^{(y)} g$ for all $N \in \mathbb{Z}_{+}$. Then one can estimate the difference $\left(w-\tilde{w}_{N}\right) \in \mathcal{X}$ subject to the norm in the Banach space $X$ :

$$
\begin{gathered}
\left\|\tilde{w}_{N}-w\right\|_{\mathcal{X}}=\left\|\tilde{w}_{N}-\mathcal{A}^{-1} g\right\|_{\mathcal{X}}=\left\|\mathcal{A}^{-1} \mathcal{A} \tilde{w}_{N}-\mathcal{A}^{-1} g\right\|_{\mathcal{X}}= \\
=\left\|\mathcal{A}^{-1}\left(\mathcal{A} \tilde{w}_{N}-g\right)\right\| \mathcal{X} \leq\left\|\mathcal{A}^{-1}\right\|\left\|\mathcal{A} \tilde{w}_{N}-g\right\|_{\tilde{\mathcal{Y}}} .
\end{gathered}
$$

Based now on inequality (5.18) we obtain that $\lim _{N \rightarrow \infty}\left\|\mathcal{A} \tilde{w}_{N}-g\right\|_{\mathcal{Y}}=0$. As the inverse operator $\mathcal{A}^{-1}$ is closed and everywhere defined and bounded, the right hand side of inequality (5.19) tends to zero as $N \rightarrow \infty$. Thereby we state that lim $\lim _{N} \| \tilde{w}_{N}-$ $w \|_{\mathcal{X}}=0$, completing the proof. $\triangleright$ 


\section{Conclusion}

We provided a precise mathematical framework for the class of control problems with delays under investigation in a Banach functional space setting, followed by careful definitions of the key properties to be analyzed such as solvability and complete controllability. The control problem was recast in a reduced form that is especially amenable to the rather innovative analytical approach that we employed. The solvability and completeness of the (reduced) nonlinearly perturbed linear control problem with delay parameters was studied in detail using a Borsuk-Ulam type fixed point theorem to analyze the general topological structure of a suitably reduced control problem solution, focused on estimating the dimension of the corresponding solution set and proving its completeness. Moreover, we investigated the related classical analytical solvability under some special, mildly restrictive, conditions imposed on the linear control and nonlinear functional perturbation. For the application of our approach, we described a new computational projection-based discretization scheme for obtaining accurate approximate solutions of the control problem along with useful error estimates. The scheme effectively reduced the infinite-dimensional problem to a sequence of solvable finite-dimensional matrix valued tasks. In addition, we included an application of the scheme to a special degenerate case wherein the Banach-Steinhaus theorem was brought to bear in the estimation process.

\section{Acknowledgements}

The authors are indebted to Professor T. Banakh for helpful remarks and instrumental discussions. Thanks are also do the Referees whose remarks and comments were useful in revising the manuscript.

\section{REFERENCES}

[1] Andres J., Górniewicz L. Topological fixed point principles for boundary value problems. Kluwer Academic Publishers, 2003

[2] Balachandran K., Byszewski L., Kim J.K. Cauchy problem for second order functional differential equations and fractional differential equations, Nonlinear Functional Analysis and Applications (2019) (in press)

[3] Banakh I., Banakh T., Plichko A., Prykarpatsky A.K. On local convexity of nonlinear mappings between Banach spaces. Cent. Eur. J. Math., 10(6), (2012), p. 2264-2271

[4] Banakh T., Lyantse W.E. , Mykytyuk Ya.V. $\infty$-Convex sets and their applications to the proof of certain classical theorems of functional analysis. Matematychni Studii. 11(1), (1999), p. 83-84

[5] Blackmore D., Prykarpatski A.K., Samolyenko V. Nonlinear dynamical systems of mathematical physics: spectral and differentialgeometric integrability analysis, World Scientific, New York, 2011

[6] Bochenek J. An abstract nonlinear second order differential equation, Ann. Polon. Math. 54.2 (1991), 155-166

[7] Byszewski L. Selected problems from the theory of differential and functional-differential equations and inequalities with nonlocal conditions, monograph of the Cracow University of Technology, Cracow, 2015 (in Polish)

[8] Dugundji J. and Granas A. Fixed point theory. PWN, Warszawa, 1981

[9] Gel'man B.D. A version of the infinite-dimensional Borsuk-Ulam theorem for multivalued maps, Sb. Math. 2016, 207, N6, p. 841

[10] Goebel K., Kirk W.A. and other (Editors) Topics in metric fixed point theory. Cambridge University Press, 1990

[11] Ioffe A.D. and Tikhomirov V.M. Theory of extremal problems. Moskow, Nauka, 1974

[12] Kato T. The theory of linear operators, NY, Springer, 1962

[13] Kolmogorov A.N., Fomin S.V. Introduction to functional analysis. Moscow, Nauka, 1987

[14] Krasnoselskiy M.A., Vainikko G.M., Zabreiko P.P. et al. Approximate solution of operator equations, Nauka, Moscow, 1969

[15] Luśtyk L., Janus J., Pytel-Kudela M., Prykarpatsky A.K. Analysis of solutions of a noncanonical Hamilton Jacobi equation using the generalized characteristics method and the Hopf Lax representations. Nonlinear Analysis 71 (2009) 5084-5089

[16] Muni V.S. Studies on controllability of dynamical systems with impulses and time-delay controls, Ph. D. Thesis, Department of Mathematics, Indian Institute of Space Science and Technology, Thiruvananthapuram, India, 2018

[17] Nirenberg L. Topics in Nonlinear functional analysis. AMS Publisher, 2001 
[18] Prykarpatski A.K. An infinite-dimensional Borsuk-Ulam-type generalization of the Leray-Schauder fixed-point theorem and some applications, Ukrainian Math. J., 60:1 2008, 114-120

[19] Prykarpatski A.K. A Borsuk-Ulam Type Generalization of the Leray-Schauder Fixed-Point Theorem, Preprint ICTP, Trieste (2007).

[20] Prykarpatski A.K. Int. Conf. “Top. Theory of Fixed and Periodic Points” (July 22-28, 2007), Held Math. Res. and Conf. Center Bedlewo, Poznań, Poland.

[21] Prykarpatski A.K. Int. Conf. "Topological Methods, Different. Equat. and Dynam. Systems”, Dedicated to the 65th Birthday of Professor Massimo Furi (June 13-16, 2007, Firenze, Italy).

[22] Prykarpatski A.K., Blackmore D. A solution set analysis of a nonlinear operator equation using a Leray-Schauder type fixed point approach, Topology 48 (2009), 182 - 185

[23] Prykarpatska N.K., Blackmore D., Prykarpatsky A.K., Pytel-Kudela M. On the inf-type exptremality solutions Hamilton-Jacobi equations, their regularity properties and some generalizations. Miskolc Mathematical Notes, Vol. 4 (2003), No. 2, pp. 157-180

[24] Rudin W. Functional analysis. McGraw-Hill Publ., International Series in Pure and Applied Mathematics, Edition: 2, 1991

[25] Saraç Y., Şener Ş.Ş. Identification of the Transverse Distributed Load in the Euler-Bernoulli Beam Equation from Boundary MeasurementInternational Journal of Modeling and Optimization, Vol. 8 (2018), No. 1, p.p.13-16

[26] Zeidler E. Nonlinear functional analysis and its applications. Springer Verlag, Berlin and Heidelberg, 1986 